\title{
A case of adverse reactions of immune thrombocytopenic purpura caused by poliovirus vaccine
}

\author{
Sumei Li, ${ }^{*}$ Yuping Zhao \\ Department of Pharmacy, The Third Affiliated Hospital of Inner Mongolia Medical University, Baotou, Inner Mongolia, China
}

Received: January 20, 2018

DOI: $10.14725 /$ dcc.v5n1p27

\author{
Accepted: February 27, $2018 \quad$ Online Published: March 10, 2018 \\ URL: http://dx.doi.org/10.14725/dcc.v5n1p27
}

\begin{abstract}
The child patient, male, 4 months and 16 days old, was vaccinated orally by Type I Type III Live Attenuated Oral Poliomyelitis Vaccine (human diploid cells) at 10:45 a.m. on June $1^{\text {st }}$ of 2016 in Shaoxian Community Health Service Center of our hospital. Two hours after vaccination, the child patient showed erythema scattered on the upper of the left knee, on the right calf and on the abdomen. The forearm of the left upper limb was scattered with punctate rashes. He was admitted to Department of Pediatrics of our hospital at 12:32 on June $1^{\text {st }}$ of 2016. The diagnosis was "immune thrombocytopenic purpura". After admission, the child patient received expert consultation and systematic treatment, consequently getting better.
\end{abstract}

Key Words: Poliovirus vaccine, Thrombocytopenic purpura, Adverse drug reaction

\section{Case information}

The child patient, male, 4 months and 16 days old, had no family history of genetic diseases and allergic reactions. He was vaccinated orally by Type I Type III Live Attenuated Oral Poliomyelitis Vaccine (human diploid cells) (Beijing Tiantan Biological Products Co., Ltd., Batch No.: 201605073, $0.1 \mathrm{ml}$ ) at 10:45 a.m. on June $1^{\text {st }}$ of 2016 in Shaoxian Community Health Service Center of our hospital. Two hours after vaccination, the child patient showed erythema scattered on the upper of the left knee, on the right calf and on the abdomen. The forearm of the left upper limb was scattered with punctate rashes. He was admitted to Department of Pediatrics of our hospital at 12:32 on June $1^{\text {st }}$ of 2016. The results of admission examination were as follows: $\mathrm{T} 36.4^{\circ} \mathrm{C}$, mental response was acceptable with sta- ble breathing. Four limbs and the trunk were scattered with visible ecchymosis and dark red petechia, which would not become shallow when pressing them. No generalized lymphadenopathy. The child patient showed flat bregma and ruddy lips. There was no obvious hyperemia in the pharynx. Bilateral lung breath sounds rough, with no obvious rhonchi and moist crackles. Heart sounds powerful, with HR 115 times/min, no murmurs. Abdomen soft with no impalpable swelling of liver and spleen. There were normal peristaltic sounds, with no positive signs in the nervous system. Routine blood tests showed WBC $7.15 \times 10^{9} / \mathrm{L}$, N $17.82 \%$, L $71.71 \%$, Hb 130.0 g/L, Plt $1.0 \times 10^{9} / \mathrm{L}$. The diagnosis was "immune thrombocytopenic purpura". After admission, the child patient received expert consultation and systematic treatment, consequently getting better. The specific therapeutic drugs were shown in Table 1.

*Correspondence: Sumei Li; E-mail: lsmstudying@163.com; Address: Department of Pharmacy, The Third Affiliated Hospital of Inner Mongolia Medical University, Baotou, Inner Mongolia, China. 
Table 1: Therapeutic drugs administered to the child patient from June $1^{\text {st }}$ to June $5^{\text {th }}$ in 2016

\begin{tabular}{lll}
\hline Therapeutic drugs & Administration Method & Administration Time \\
\hline I.V. Human Immunoglobulin $7 \mathrm{~g}+0.9 \%$ Sodium Chloride Injection $50 \mathrm{ml}$ & ivd, qd & $1-2$ Days \\
Methylprednisolone Sodium Succinate for Injection $15 \mathrm{mg}+5 \%$ Glucose Injection $20 \mathrm{ml}$ & ivd, qd & $1-3$ Days \\
Etamsylate Injection $0.0625 \mathrm{~g}+5 \%$ Glucose Injection $20 \mathrm{ml}$ & ivd, q8h & I-4 Days \\
Vitamin $\mathrm{K}_{1}$ Injection $5 \mathrm{mg}+5 \%$ Glucose Injection $20 \mathrm{ml}$ & $1-5$ Days \\
\hline
\end{tabular}

On June $6^{\text {th }}$, the child patient showed normal body temperature, and the original petechia and ecchymosis were slightly relieved, with no fresh rashes. Besides, he showed no symptoms of gingival bleeding, epistaxis, haematemesis, melena, hematuria and other bleeding tendencies. He could take in milk and sleep well. The condition of defecation and urination was normal. The results of physical examination were as follows: mental responses were acceptable with stable breathing. Four limbs and the trunk were scattered with visible old ecchymosis and dark red petechia, which would not become shallow when pressing them. No generalized lymphadenopathy, with flat bregma. Re-examination results of routine blood tests showed: WBC $6.42 \times 10^{9} / \mathrm{L}, \mathrm{N}$ $34.92 \%$, L $61.81 \%$, Hb 115.0 g/L, Plt $440.0 \times 10^{9} / \mathrm{L}$. No abnormalities were observed in cell morphology. The patient was admitted to leave hospital with the condition improved. He was required to receive routine blood tests through outpatient review, as well as periodical follow-up. Upon being discharged from inpatient care, the child patient needed to take $5 \mathrm{mg} / \mathrm{d}$ prednisone (tablets) for oral administration. Symptoms were gradually resolved after 1 week, the dosage of prednisone (tablets) were reduced to $2.5 \mathrm{mg} / \mathrm{d}$, and symptomatic treatment was given for 1 week. In the follow-up visit after one month, the child patient showed no ecchymosis and dark red petechia, with the normality in the routine blood tests.

\section{Discussion}

According to National Immunization Program, poliovirus vaccine is classified as one of A-class vaccines. It is a type of free vaccine provided by the government to infants and kids at 2 months, 3 months, 4 months and 4 years of age (the first three times for oral vaccination). This vaccine is mainly used to prevent poliomyelitis caused by poliovirus. According to the vaccine instructions, this product is generally free of side effects after oral administration. Some individuals have fever, nausea, emesis, diarrhea and rashes. Therefore, "immune thrombocytopenic purpura" caused by Type I Type III Live Attenuated Oral Poliomyelitis Vaccine (human diploid cells) is a new adverse reaction. In the survey after this incident, this kind of phenomenon was not found to happen to other kids who were orally vaccinated by vaccines of the same batch in our community or other communities.

It has been reported that injectable poliovirus vaccine ${ }^{[1]}$ and poliovirus vaccine simultaneously vaccinated with other vaccines, ${ }^{[2]}$ may cause rashes and induce "idiopathic thrombocytopenic purpura". Shen XM et al. ${ }^{[3]}$ once reported a case of a child patient having idiopathic thrombocytopenic purpura after oral vaccination of poliovirus vaccine. No more reports were found since then. Therefore, this case was the first case reported in recent 15 years that a patient was newly diagnosed with "immune thrombocytopenic purpura" caused by only oral vaccination of live attenuated oral poliomyelitis vaccine, it was also the first case that a patient had "immune thrombocytopenic purpura" caused by Type I Type III Live Attenuated Oral Poliomyelitis Vaccine (human diploid cells), which should be noted accordingly. At the same time, the author looked up "Practice of Pediatrics" [4] to determine the causes of "idiopathic thrombocytopenic purpura" and its pathogenesis, finding that " 1 percent of cases arise from the onset of vaccination". On account of that, it is necessary to pay more attention to the safety of drug usage about Type I Type III Live Attenuated Oral Poliomyelitis Vaccine (human diploid cells).

In summary, in order to use Type I Type III Live Attenuated Oral Poliomyelitis Vaccine (human diploid cells) more safely and reduce the incidence of adverse reactions after vaccination, the following two points should be noted in the application of vaccines: (1) Before using the vaccine, the doctor should inquire about the child patient's past medical history and allergic history; it should be prudent to vaccinate children with allergy to drugs and some food; (2) The safety about the vaccination process should be noticed with 30minute on-site observation after vaccination required, and health care personnel were required to carefully monitor child patient's mental status and physical responses. At the same time, once such adverse reactions occur in the application of such vaccines, it is required to cope with it in a prompt and timely way. Send child patients to a higher-level hospital for hospitalization if necessary. In short, since infants and kids are all special groups, we must be alert to the occurrence of the physical damages caused by vaccination.

\section{Conflicts of Interest Disclosure}

The authors have no conflicts of interest related to this article. 


\section{References}

[1] He XZ, Bai SX, Fu Y, et al. A case of immune thrombocytopenia caused by imported live attenuated oral poliomyelitis vaccine. Herald of Medicine. 2015; 34(6): 755-756.

[2] Wang YL, Fang F, Chen GJ, et al. A case of idiopathic thrombocytopenic purpura caused by simultaneous vaccination of live attenu- ated measles vaccine and live attenuated oral poliomyelitis vaccine. Chinese Journal of Vaccines and Immunization. 2008; 14(4): 334.

[3] Shen XM, Yang TH. A case of idiopathic thrombocytopenic purpura caused by oral vaccination of live attenuated poliomyelitis vaccine. Chinese Journal of Vaccines and Immunization. 2002; 8(3): 153.

[4] Zhu FT. Practice of Practice of Pediatrics. Eighth Edition. Beijing: People's Medical Publishing House; 2015. 1759-1760 p. 